\title{
Mamíferos do município de Fênix, Paraná, Brasil: etnozoologia e conservação
}

\author{
Fabiana Rocha-Mendes ${ }^{1,2}$, Sandra B. Mikich ${ }^{3}$, Gledson V. Bianconi ${ }^{2} \&$ Wagner A. Pedro ${ }^{4}$ \\ 1 Programa de Pós-graduação em Biologia Animal, Universidade Estadual Paulista. Rua Cristóvão Colombo 2265, \\ 15054-000 São José do Rio Preto, São Paulo, Brasil.E-mail: gabi_frm@terra.com.br \\ ${ }^{2}$ Mülleriana: Sociedade Fritz Müller de Ciências Naturais. Caixa Postal 19093, 81531-980 Curitiba, Paraná, Brasil. \\ ${ }^{3}$ Laboratório de Ecologia, Embrapa Florestas. Caixa Postal 319, 83411-000 Colombo, Paraná, Brasil. \\ E-mail: sbmikich@cnpf.embrapa.br \\ ${ }^{4}$ Laboratório de Chiroptera, Departamento de Apoio, Produção e Saúde Animal, Universidade Estadual Paulista. \\ Caixa Postal 341, 16050-680 Araçatuba, São Paulo, Brasil. E-mail: wpedro@fmva.unesp.br
}

\begin{abstract}
Mammals of the municipality of Fênix, Paraná, Brazil: ethnozoology and conservation. Based on an ethnozoological approach we obtained historical and present information on the mammalian fauna of the municipality of Fênix, located in the mid-western Paraná state, Southern Brazil. To do so, in 2004 we interviewed 19 local residents. The interview consisted of an informal talk based on a questionary followed by the presentation of photographs of potential mammalian species of the study region. As a result, we recorded 39 species, of which at least six are new records for the study area. Hunting revealed to be not only common in the past, but also still practiced nowadays, even inside nature preserves, like the Parque Estadual Vila Rica do Espírito Santo. The species more appreciated by the hunters are the same cited for other neotropical regions. At present, however, Hydrochaeris hydrochaeris (Linnaeus, 1766) (Hydrochaeridae) is the species more frequently sought since it is still common in several natural areas of the region, including those that have been largely modified by man. With regard to mammalian predation upon domestic animals, almost $80 \%$ of the interviewees declared that they had lost some breed, especially chicken, as the result of the attack of medium-sized carnivores. Data on temporal modifications of the mammalian species composition were also obtained with the interviews, as the local extinctions of some large mammals (Panthera onca (Linnaeus, 1758) (Felidae), Tapirus terrestris (Linnaeus, 1758) (Tapiridae) and Tayassu pecari (Link, 1795) (Tayassuidae)) or the population increases of Cebus nigritus (Goldfuss, 1809) (Cebidae) and Nasua nasua (Linnaeus 1766) (Procyonidae), which are supposedly related to the consumption of corn and other plantations.

KEY WORDS. Endangered species, forest fragmentation, hunting, interviews, mammalian inventory.
\end{abstract}

RESUMO. Com base na abordagem etnozoológica foram resgatadas informações históricas e atuais sobre a mastofauna do município de Fênix, mesorregião centro-ocidental do estado do Paraná, sul do Brasil. Para tanto, no ano de 2004, foram realizadas entrevistas com 19 moradores locais por meio de uma conversa informal que tinham por objetivo o preenchimento de um questionário básico e a apresentação de fotografias da fauna potencial da região. Como resultado, foram registradas 39 espécies de mamíferos, sendo que destas, pelo menos seis são novos registros para a área. Informações obtidas em relação à caça indicam que esta atividade, muito comum no passado, ainda é presente, inclusive em unidades de conservação, como o Parque Estadual Vila Rica do Espírito Santo. As espécies que sofreram e sofrem maior pressão de caça são as mesmas citadas para outras partes do neotrópico. Atualmente, Hydrochaeris hydrochaeris (Linnaeus, 1766) (Hydrochaeridae) é a espécie mais procurada, dada à facilidade de encontrála em diversas áreas naturais ou parcialmente antropizadas do município. No que se refere à predação causada por animais silvestres sobre animais domésticos, quase $80 \%$ dos entrevistados relataram ter sofrido perdas em suas criações, principalmente galinhas, resultantes de ataques de carnívoros de médio porte. Dados sobre alterações temporais na composição da mastofauna também foram obtidos junto aos entrevistados, como a extinção local de alguns mamíferos de grande porte (Panthera onca (Linnaeus, 1758) (Felidae), Tapirus terrestris (Linnaeus, 1758) (Tapiridae) e Tayassu pecari (Link, 1795) (Tayassuidae)) e o aumento populacional de Cebus nigritus (Goldfuss, 1809) (Cebidae) e de Nasua nasua (Linnaeus 1766) (Procyonidae), supostamente relacionado ao consumo de plantações como o milho. PALAVRAS CHAVE. Caça, entrevistas, espécies ameaçadas, fragmentação florestal, inventário mastofaunístico. 
O prefixo ethno tem sido freqüentemente utilizado por significar, de maneira sintetizada, os modos que as sociedades compreendem o mundo (MarTin 1995). Assim, o termo etnobiologia significa "forma com que as diferentes populações humanas percebem, classificam e entendem os recursos naturais" (CLÉMENT 1998); quando o prefixo ethno é usado seguido do nome de uma disciplina acadêmica, como a biologia ou a zoologia, dá a entender que os pesquisadores dessas áreas estão buscando as percepções de sociedades locais dentro desses contextos (HaverRoth 1997).

Nos últimos anos, um número crescente de estudos etnozoológicos tem sido realizado no Brasil, especialmente entre as populações indígenas da região Amazônica (e.g. JeNSEN 1988, BEGOssi 1999). O principal interesse tem sido aquele relacionado às questões lingüísticas, isto é, de que forma os grupos nomeiam e categorizam sua fauna familiar.

Distinta, porém igualmente preciosa, é a etnozoologia como ferramenta interpretativa do histórico compartilhado entre homens e animais em uma determinada região. Aqui, incluem-se diferentes manifestações humanas frente à fauna, sejam estas inspiradas pela afeição, repúdio, reverência ou desprezo, indicando, por vezes, crendices e aspectos cinegéticos locais. Com ela, ora obtêm-se, ora resgatam-se informações bionômicas relevantes à conservação e/ou restauração de seus bens naturais. Para contextualizar podemos citar trabalhos realizados com as populações não-indígenas do Sudeste e Sul brasileiros, conforme segue.

Para o estado de São Paulo, Cullen Jr. et al. (2000) enfocaram a pressão da caça sobre os mamíferos e aves em fragmentos florestais; no estado do Paraná, Azevedo \& Conforti (2002) realizaram um estudo sobre a percepção das comunidades do entorno do Parque Nacional do Iguaçu, em relação aos grandes felinos; e Pedroso Jr. \& SATo (2003) trabalharam, por meio da educação ambiental, a percepção da fauna terrestre no Parque Nacional de Superagüi.

Considerando a súbita ocupação de muitas regiões paranaenses, onde a destruição de grandes áreas florestais ocorreu com tal velocidade que muitas informações preciosas sobre sua mastofauna foram perdidas, os estudos etnozoológicos podem ser uma importante ferramenta no resgate desses dados.

A mesorregião centro-ocidental do Paraná, onde se insere a área de estudo deste trabalho, merece especial atenção nesse sentido. Em conseqüência de seus solos (a "terra roxa") de ótima qualidade para a agricultura, a região sofreu uma ocupação e um desmatamento bastante intensos (MıкICH \& SiLVA 2001, Mikich \& Oliveira 2003).

Com base nesta problemática, o presente estudo objetivou resgatar informações históricas e atuais da mastofauna de Fênix - Paraná, analisar a relação do homem com os mamíferos da região e levantar aspectos bio-ecológicos para algumas espécies.

\section{MATERIAL E MÉTODOS}

\section{Área de estudo}

O município de Fênix localiza-se na mesorregião centroocidental (IPARdes 2004) do Paraná, Sul do Brasil, entre os rios
Ivaí e Piquiri. O relevo é levemente ondulado e a altitude média é de 650 m (Мikich \& Oliveira 2003). O clima da região, segundo classificação de Köppen, é do tipo Cfa ou subtropical úmido mesotérmico, com verões quentes e geadas pouco freqüentes. Há tendência de concentração de chuvas entre os meses de dezembro e fevereiro, e o período mais seco do ano ocorre entre os meses de junho e agosto (MAAck 1981, Мiкich \& Oliveira 2003). Considerando aspectos fitogeográficos, Fênix está inserida no bioma Floresta Atlântica, mais especificamente nos domínios da Floresta Estacional Semidecidual (Veloso et al. 1991, Мikich \& SiLVA 2001).

Com uma área de aproximadamente $224 \mathrm{~km}^{2}$, Fênix $\left(23^{\circ} 54^{\prime} \mathrm{S}\right.$ e $\left.51^{\circ} 58^{\prime} \mathrm{W}\right)$ possui, segundo IBge (2004), 4.942 habitantes, a maioria (78\%) vivendo no meio urbano, sendo as culturas de milho e soja a principal fonte de renda (Miкich \& OLIVEIRA 2003).

Embora o município tenha sido criado em 25 de julho de 1960 (Lei Estadual 4245), os primórdios históricos de sua ocupação remontam ao século XVI, quando a região, então habitada por índios guaranis, foi palco para a base das operações espanholas em terras guairenhas, com a formação da vila espanhola Villa Rica del Espiritu Santo (1592-1632) (PARellada 1993).

Após Villa Rica ter sido sitiada, destruída e incendiada em 1632, quase 300 anos se passaram para que a região, de fato, voltasse a se desenvolver (FerReira 1999). A boa localização do município (próxima a dois grandes rios: Ivaí e Corumbataí), aliada à alta produtividade de suas terras, propiciou um rápido desenvolvimento econômico e, conseqüentemente, seu acelerado desmatamento (Mikich \& Oliveira 2003, Paranacidade 2004).

Como resultado da abrupta ocupação, a configuração florestal atual do município apresenta poucos fragmentos, de pequenas extensões e com diferentes graus de alteração. Além disso, a maior parte das florestas ciliares, que poderiam atuar como corredores naturais para a fauna, foi drasticamente alterada, reduzida ou inteiramente substituída por cultivos agrícolas e pastagens (Мiкich \& Silva 2001, Мiкich et al. 2004).

Embora informações de todo o município fossem de interesse deste trabalho, para a obtenção de dados sobre a situação atual da mastofauna local foram selecionados três remanescentes florestais localizados próximos à sede do município. Estes foram escolhidos com base nos diversos estudos zoológicos ali realizados, permitindo assim discussões sobre as informações obtidas.

São eles: 1) o Parque Estadual Vila Rica do Espírito Santo (VR) $\left(23^{\circ} 55^{\prime} \mathrm{S}\right.$ e $\left.51^{\circ} 57^{\prime} \mathrm{W}\right)$, que possui 354 ha e tem como limites os rios Ivaí e Corumbataí e áreas de monoculturas; 2) a Fa-

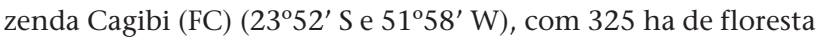
nativa dividida em duas porções não conectadas, limitadas por monoculturas e pelo rio Ivaí; e 3) a Fazenda Guajuvira (FG) $\left(23^{\circ} 53^{\prime} \mathrm{S}\right.$ e $\left.51^{\circ} 57^{\prime} \mathrm{W}\right)$, localizada entre as outras duas áreas acima citadas, possuindo somente 24 ha de floresta, dividida em duas porções de tamanhos regulares cujos limites também são dados por áreas cultivadas e pelo rio Ivaí (Mikich \& Silva 2001). 


\section{Entrevista com a população}

Entre os dias 23 e 25 de fevereiro de 2004, foram realizadas entrevistas com 19 moradores de Fênix. Os entrevistados foram escolhidos com base nos seguintes critérios: 1) proximidade de suas moradias com os remanescentes florestais estudados; 2) período de residência no município, favorecendo os moradores mais antigos; 3 ) ocupação profissional, com preferência por funcionários das áreas florestais de estudo, e 4) indicação por terceiros, especialmente de caçadores e/ou pescadores.

As entrevistas foram realizadas através de uma conversa informal que tinha por objetivo o preenchimento de um questionário básico, além da apresentação de fotografias de alguns mamíferos de provável ocorrência na região para que houvesse a confirmação ou não de sua presença, bem como a citação do nome pelo qual cada espécie é conhecida localmente.

Para classificar as espécies mencionadas nas entrevistas em relação à sua popularidade na área de estudo, foi aplicado o Índice de Constância (Silveira-Neto et al. 1976), onde, as espécies citadas em mais de 50\% das entrevistas foram tidas como constantes, de 25 a 50\% como acessórias e em menos de $25 \%$ como ocasionais. Para a análise dos demais dados obtidos, foram utilizadas porcentagens.

\section{RESULTADOS}

\section{Caracterização das entrevistas}

Todos os 19 entrevistados eram do sexo masculino, embora algumas vezes esposas e filhas estivessem presentes e também contribuíssem com informações. A idade média dos entrevistados foi de 53 anos, variando de 36 a 79 anos, e o tempo médio de residência em Fênix foi de 38 anos, com mínimo de 1 ano e máximo de 54 anos. O tempo médio de duração das entrevistas foi de 38 minutos, variando entre 15 e 77 minutos.

Todos os moradores de residências próximas às unidades florestais bases deste estudo (VR, FC e FG), bem como os funcionários destas áreas foram entrevistados. Assim, o número de entrevistados $(n=19)$ pode ser considerado satisfatório para a obtenção das informações almejadas. Este número representa $0,4 \%$ da população do município $(n=4.942)$ e $1 \%(n=1.955)$ dos moradores do sexo masculino acima de 10 anos (IbGE 2004).

\section{Levantamento de espécies}

Foram registradas 39 espécies distribuídas em 19 famílias e oito ordens (Tab. I). A classificação das espécies em ocasionais, acessórias ou constantes nas entrevistas, bem como os nomes pelos quais são localmente conhecidas, constam na tabela I. O ordenamento taxonômico segue o proposto por WILSON \& REEDER (1993).

Embora os dados coletados sobre os veados do gênero Mazama Rafinesque, 1817 (Cervidae) tenham permitido o registro de pelo menos três espécies: M. nana (Hensel, 1872), M. americana (G. Fischer, 1814) e M. gouazoupira (G. Fischer, 1814), suas identificações em nível específico não ocorreram em todas as entrevistas. Para os pequenos gatos-do-mato do gênero
Leopardus Gray, 1842 (Felidae), somente L. wiedii (Schinz, 1821) foi identificado como um táxon distinto, em uma entrevista; nas demais, L. wiedii e L. tigrinus (Schreber, 1775) foram considerados uma única espécie. Deste modo, para os veados e estas duas espécies de felinos, as informações foram apresentadas em nível genérico (Tab. I), embora a presença da maioria delas tenha sido confirmada em campo pelos autores.

\section{Sobre a caça}

Oitenta e quatro porcento dos entrevistados declaram ter feito o consumo de animais silvestres, enquanto, somente $26 \%$ admitiram a efetiva realização da caça por esporte e/ou alimentação. As espécies citadas pelos caçadores como normalmente procuradas, por ordem decrescente de importância foram: Mazama spp., Tayassu pecari (Link, 1795) (Tayassuidae), Tapirus terrestris (Linnaeus, 1758) (Tapiridae), Agouti paca (Linnaeus, 1766) (Agoutidae), Pecari tajacu (Linnaeus, 1758) (Tayassuidae), Nasua nasua (Linnaeus, 1766) (Procyonidae) e Hydrochaeris hydrochaeris (Linnaeus, 1766) (Hydrochaeridae). Todavia, quando lhes foi perguntado os animais mais procurados para caça na região, a ordem de importância foi alterada para: $H$. hydrochaeris, A. paca, Mazama spp., N. nasua, Cebus nigritus (Goldfuss, 1809) (Cebidae), P. tajacu, Dasypus spp. (Dasypodidae), T. terrestris, Myrmecophaga tridactyla Linnaeus, 1758 (Myrmecophagidae), Dazyprocta azarae Lichtenstein, 1823 (Dasyproctidae) e Alouatta guariba (Humboldt, 1812) (Atelidae).

A caça de Pteronura brasiliensis (Gmelin, 1788) (Mustelidae), Panthera onca (Linnaeus, 1758) (Felidae) e Leopardus pardalis (Linnaeus, 1758) (Felidae) também foi relatada, estando principalmente relacionada à retaliação ao ataque de animais domésticos, embora, em todos os casos, a carne destes animais tenha sido aproveitada para consumo.

De acordo com as descrições apresentadas pelos entrevistados, as técnicas de caça variam conforme a espécie-alvo. No caso da capivara (H. hydrochaeris), esta é feita com o uso de barcos, faroletes e espingardas, podendo também envolver o uso de uma rede colocada dentro do rio para interceptar o nado do animal e obrigá-lo a emergir no local esperado pelo caçador. Os locais mais utilizados para a caça dessa espécie são os rios, principalmente aqueles localizados nas divisas do VR (rios Ivaí e Corumbataí) e os arrozais próximos à cursos d'água.

Para a caça de veados (Mazama spp.) são utilizados cães "do tipo americano" e espingardas. Há preferência pela caça esportiva destes animais por proporcionar maior divertimento - atividade localmente conhecida como a "corrida dos cachorros". Segundo alguns caçadores, antes do desmatamento qualquer área florestada tinha muitos veados, sendo estes amplamente caçados. Hoje, porém, as áreas mais utilizadas são as fazendas Cagibi e Valadão.

A caça da paca (A. paca) emprega jiraus, cevas de milho ou armadilhas. Já a anta (T. terrestris) é caçada nos rios, com o auxílio de cachorros ou utilizando a técnica da "espera".

Sobre os locais ainda utilizados para a caça na região, 37\% dos entrevistados citaram as imediações e o interior do VR. Cabe 
Tabela I. Lista, classes de ocorrência e status das espécies de mamíferos do município de Fênix, Paraná, com base em entrevistas realizadas em 2004 com 19 moradores locais.

\begin{tabular}{|c|c|c|}
\hline Táxons & Nomes populares locais & Ocorrência \\
\hline \multicolumn{3}{|l|}{ Didelphimorphia } \\
\hline \multicolumn{3}{|l|}{ Didelphidae } \\
\hline Chironectes minimus (Zimmermann, 1780) ${ }^{3}$ & Cuíca-d'água & Ocasional \\
\hline Didelphis albiventris Lund, 1840 & Gambá & Ocasional \\
\hline \multicolumn{3}{|l|}{ Xenarthra } \\
\hline \multicolumn{3}{|l|}{ Dasypodidae } \\
\hline Cabassous tatouay (Desmarest, 1804) & Tatu-do-rabo-mole & Acessória \\
\hline Dasypus novemcinctus Linnaeus, 1758 & Tatu-galinha & Constante \\
\hline Dasypus septemcinctus Linnaeus, $1758^{4}$ & Tatu-bolinha & Acessória \\
\hline Euphractus sexcinctus (Linnaeus, 1758) & Tatu-testa, tatu-peba & Constante \\
\hline \multicolumn{3}{|l|}{ Myrmecophagidae } \\
\hline Myrmecophaga tridactyla Linnaeus, $1758^{3}$ & Tamanduá-bandeira & Ocasional \\
\hline Tamandua tetradactyla (Linnaeus, 1758) & Tamanduá & Constante \\
\hline \multicolumn{3}{|l|}{ Primates } \\
\hline \multicolumn{3}{|l|}{ Atelidae } \\
\hline Alouatta guariba (Humboldt, 1812) ${ }^{4}$ & Bugio & Ocasional \\
\hline \multicolumn{3}{|l|}{ Cebidae } \\
\hline Cebus nigritus (Goldfuss, 1809) & Macaco-prego & Constante \\
\hline \multicolumn{3}{|l|}{ Carnivora } \\
\hline \multicolumn{3}{|l|}{ Canidae } \\
\hline Cerdocyon thous (Linnaeus, 1766) & Cachorro-do-mato, raposa & Constante \\
\hline Pseudalopex gymnocercus (G. Fischer, 1814) ${ }^{4}$ & Cachorro-do-mato & Ocasional \\
\hline \multicolumn{3}{|l|}{ Felidae } \\
\hline Herpailurus yaguarondi (Lacépède, 1809) & Mourisco, mourício, canguçu & Constante \\
\hline Leopardus pardalis (Linnaeus, 1758) & Jaguatirica & Constante \\
\hline Leopardus spp. ${ }^{1}$ & Gato-do-mato-pintado, mamoninha & Constante \\
\hline Puma concolor (Linnaeus, 1771) & Puma & Constante \\
\hline Panthera onca (Linnaeus, 1758) ${ }^{3}$ & Onça-pintada & Acessória \\
\hline \multicolumn{3}{|l|}{ Mustelidae } \\
\hline Lontra longicaudis (Olfers, 1818) & Lontra & Constante \\
\hline Pteronura brasiliensis (Gmelin, 1788) ${ }^{4}$ & Ariranha & Constante \\
\hline Conepatus chinga (Molina, 1782) ${ }^{4}$ & Zorrilho & Ocasional \\
\hline Eira barbara (Linnaeus, 1758) & Irara & Constante \\
\hline Galictis cuja (Molina, 1782) & Furão, jaratataca, guaripeva & Constante \\
\hline \multicolumn{3}{|l|}{ Procyonidae } \\
\hline Nasua nasua (Linnaeus 1766) & Quati, quati-mundéo & Constante \\
\hline Procyon cancrivorus (G. Cuvier, 1798) & Mão-pelada, quatizão, guará, cachorro-do-mato, guaxinim & Constante \\
\hline \multicolumn{3}{|l|}{ Perissodactyla } \\
\hline \multicolumn{3}{|l|}{ Tapiridae } \\
\hline Tapirus terrestris (Linnaeus, 1758) ${ }^{3}$ & Anta & Acessória \\
\hline \multicolumn{3}{|l|}{ Artiodactyla } \\
\hline \multicolumn{3}{|l|}{ Tayassuidae } \\
\hline Pecari tajacu (Linnaeus, 1758) ${ }^{3}$ & Queixada & Constante \\
\hline Tayassu pecari (Link, 1795) & Cateto & $\begin{array}{c}\text { Constante } \\
\text { Continua }\end{array}$ \\
\hline
\end{tabular}


Tabela I. Continuação.

\begin{tabular}{|c|c|c|}
\hline Táxons & Nomes populares locais & Ocorrência \\
\hline \multicolumn{3}{|l|}{ Cervidae } \\
\hline Mazama spp. ${ }^{2}$ & Veado, guatapará, cambuta, vermelho, mateiro, pardo, catingueiro & Constante \\
\hline \multicolumn{3}{|l|}{ Rodentia } \\
\hline \multicolumn{3}{|l|}{ Sciuridae } \\
\hline Sciurus aestuans Linnaeus, 1766 & Serelepe & Ocasional \\
\hline \multicolumn{3}{|l|}{ Erethizontidae } \\
\hline Sphiggurus spinosus (F. Cuvier, 1823) & Ouriço & Acessória \\
\hline \multicolumn{3}{|l|}{ Caviidae } \\
\hline Cavia aperea Erxleben, 1777 & Preá & Ocasional \\
\hline \multicolumn{3}{|l|}{ Hydrochaeridae } \\
\hline Hydrochaeris hydrochaeris (Linnaeus, 1766) & Capivara & Constante \\
\hline \multicolumn{3}{|l|}{ Dasyproctidae } \\
\hline Dasyprocta azarae Lichtenstein, 1823 & Cutia & Acessória \\
\hline \multicolumn{3}{|l|}{ Agoutidae } \\
\hline Agouti paca (Linnaeus, 1766) & Paca & Constante \\
\hline \multicolumn{3}{|l|}{ Lagomorpha } \\
\hline \multicolumn{3}{|l|}{ Leporidae } \\
\hline Lepus europaeus Pallas, 1778 & Lebre & Ocasional \\
\hline Sylvilagus brasiliensis (Linnaeus, 1758) & Tapeti & Ocasional \\
\hline
\end{tabular}

ressaltar que um dos entrevistados acrescentou que esta unidade de conservação apresenta fiscalização inadequada.

Adicionalmente, um dos entrevistados relatou que, em meados de 1977, moradores do município de Jacarezinho (Norte pioneiro do Paraná) vinham à Fênix para caçar e comprar peles de animais silvestres. Nestas circunstâncias, alguns moradores locais matavam os animais para consumo e guardavam as peles para venda. As espécies mais procuradas pelos compradores eram: gatos-do-mato (Leopardus spp. e Herpailurus yaguarondi (Lacépède, 1809) (Felidae)), porcos-do-mato (T. pecari e P. tajacu), veados (Mazama spp.) e cuíca d'água (Chironectes minimus (Zimmermann, 1780) (Didelphidae)).

\section{Ataques a criações domésticas}

O ataque de carnívoros silvestres a criações domésticas foi relatado em cerca de $79 \%$ das entrevistas, sendo os mais atacados: galinhas (citadas por 100\% dos entrevistados), ovos (27\%), gatos domésticos (7\%), patos (7\%), perus (7\%) e porcos (7\%).

A identificação do animal responsável pelo ataque foi realizada por eles com base na visualização do evento, rastros, sondagem, captura com armadilhas e abate dos predadores. Segundo um dos entrevistados, casos de predação a animais domésticos por onça-pintada não eram comuns, uma vez que haviam muitas presas naturais disponíveis quando a espécie ocorria freqüentemente na região.
Os carnívoros tidos como responsáveis pelos eventos de predação foram: Leopardus spp., H. yaguarondi, Cerdocyon thous (Linnaeus, 1766) (Canidae) (citados em 33\% dos casos), N. nasua (20\%), Procyon cancrivorus (G. Cuvier, 1798) (Procyonidae) (13\%), Eira barbara (Linnaeus, 1758) (Mustelidae) (13\%), Galictis cuja (Molina, 1782) (Mustelidae) (13\%), L. pardalis (6\%) e P. onca (6\%). Foi relatado que as investidas foram principalmente a animais criados soltos, embora ataques a galinheiros por cachorros-domato também tenham sido observados. Segundo alguns entrevistados, o alto número de eventos de predação pode estar relacionado ao não confinamento das criações. Além disso, a utilização de cachorros domésticos para proteção da criação foi indicada como um método eficaz de prevenção aos ataques.

\section{Alterações temporais na composição da mastofauna}

Setenta e quatro porcento dos entrevistados acreditam que antigamente havia uma maior quantidade de mamíferos na região, podendo seu decréscimo, em riqueza e abundância, estar relacionado à caça, ao desmatamento, à predação por cachorros domésticos e a uma severa tempestade que atingiu a região no ano de 1995 . Por outro lado, 21\% acreditam que atualmente existem mais animais devido à diminuição da caça, ao aumento da fiscalização e ao desmatamento, que resultou na concentração de grande parte da mastofauna silvestre nos poucos fragmentos florestais existentes na região, como o VR. So- 
mente um entrevistado (5\%) não manifestou sua opinião sobre o assunto.

Adicionalmente, 50\% dos entrevistados relataram ter havido um aumento populacional de C. nigritus, N. nasua e também, embora em menor proporção, de $P$. tajacu, estando este fato relacionado à utilização das plantações (soja e, principalmente, milho) como fonte de alimento.

\section{DISCUSSÃO}

\section{Levantamento de espécies}

Diversos métodos podem ser empregados na obtenção de uma lista de mamíferos para uma determinada região, mesmo que estes não ocorram mais na área. Dentre os mais utilizados, podemos citar: consultas bibliográficas e museológicas; capturas com gaiolas, armadilhas-de-queda (pitfall) e redes-deneblina; busca de evidências diretas (p.ex. visualizações, vocalizações) ou indiretas (p.ex. pegadas e fezes); e, mais recentemente, armadilhas fotográficas (Voss \& EMmons 1996, Wilson et al. 1996). Além desses, entrevistas com moradores da área de estudo pode ser considerado um método alternativo, de baixo custo, que pode ser realizado em menor tempo que os demais, e que gera informações não só das espécies ocorrentes na área (FONSECA 2001), mas também de aspectos etnozoológicos e bionômicos relacionados a estas (e.g. Vidolin et al. 2002, Conforti \& Azevedo 2003, Pedroso Jr. \& Sato 2003, este estudo).

Embora limitada para o inventariamento de pequenos mamíferos (Rodentia, Didelphimorphia e Chiroptera), esta técnica pode ser considerada eficaz no registro de espécies de médio e grande porte, já que esses grupos, em geral, possuem hábito noturno ou crepuscular, áreas de vida relativamente grandes e baixas densidades, o que dificulta seu levantamento por meio de métodos tradicionais (PARDini et al. 2003). Cabe salientar, no entanto, que um inventário mastofaunístico satisfatório deve contemplar a aplicação de variadas técnicas de amostragem, permitindo assim uma maior integração das informações disponibilizadas e, conseqüentemente, uma listagem segura das espécies ocorrentes numa determinada área (Voss \& EmMons 1996).

Até o momento, Fênix foi contemplada com duas listas de mamíferos. A primeira, apresentada no plano de manejo do Parque Estadual Vila Rica do Espírito Santo (ITCF 1987), conta com uma relação simples, com 14 espécies de médio e grande porte. A segunda, resultado da revisão do plano de manejo desta unidade de conservação, apresentou uma listagem de 44 táxons, incluindo os grupos de pequenos mamíferos, não apreciados na primeira edição (Mikich \& Oliveira 2003).

A lista apresentada neste trabalho, baseada apenas nas entrevistas com a comunidade local, relaciona 39 espécies, sendo que 14 são possíveis novos registros para a área, pois foram registradas exclusivamente por este método. São elas:

Chironectes minimus: espécie de marsupial de hábito semi-aquático com distribuição em todo território paranaense ( $v$. LANGE \& Jablonski 1998, Eisenberg \& Redford 1999). Possui hábito noturno e solitário (EMmONs 1997) e atualmente encontra-se na lista de espécies ameaçadas do Paraná (Margarido \& Braga 2004). É considerado de difícil visualização, o que explica, em hipótese, sua ausência nas listas pré-existentes para área. Cabe mencionar, entretanto, que a citação da espécie data do final da década de 1970, podendo em dias atuais estar localmente extinta.

Cabassous tatouay (Desmarest, 1804) (Dasypodidae): apesar de poucos registros para o estado, a distribuição deste tatu se dá para áreas de Floresta Estacional Semidecidual, Floresta Ombrófila Mista, Floresta Ombrófila Densa e Campos Naturais (Margarido \& Braga 2004). Recentemente um exemplar da espécie foi coletado em Fênix e depositado na coleção de mamíferos do Museu de História Natural Capão da Imbuia, Curitiba (Margarido \& Braga 2004).

Dasypus septemcinctus Linnaeus, 1758 (Dasypodidade): este tatu ocorre nos Campos Naturais, Floresta Estacional Semidecidual e Floresta Ombrófila Mista (MARgarido \& Braga 2004). Embora registrado em quase 50\% das entrevistas, sua presença em Fênix merece ser investigada, pois a semelhança morfológica da espécie com o tatu-galinha (D. novemcinctus Linnaeus, 1758) (Dasypodaidae) pode resultar em erros de identificação.

Euphractus sexcinctus (Linnaeus, 1758) (Dasypodidae): esta espécie de tatu é encontrada principalmente em ambientes abertos; sua ocorrência está associada aos Campos Naturais e ao Cerrado ( $v$. EIsENberg \& REDFord 1999). O registro para a área de estudo ocorreu em $63 \%$ das entrevistas, sendo inclusive obtido junto a um dos entrevistados a cauda de um exemplar recentemente morto por cães.

Myrmecophaga tridactyla: a área de ocorrência do tamanduábandeira abrange principalmente os Campos Naturais e o Cerrado, sendo escassos os registros para as formações florestais (Margarido \& Braga 2004). Porém, Bigg-Wither (1974), em sua expedição pelo vale do rio Ivaí (1872-1875), afirma ter visto e matado vários tamanduás-bandeira em áreas florestadas da região. Dois entrevistados relataram a presença da espécie para o município, sendo o mais recente o de um animal visualizado em área alterada, no ano de 2003.

Alouatta guariba: este primata ocorre em todo o território paranaense (Margarido \& Braga 2004). Foi citado em apenas $10 \%$ das entrevistas, sendo, porém, de ocorrência improvável para o VR e remanescentes florestais próximos, já que a espécie possui vocalização característica que pode ser ouvida a longas distâncias. Este fato, aliado às pequenas dimensões dos fragmentos florestais estudados e aos exaustivos inventários mastofaunísticos ali realizados, tornam remotas suas chances de ocorrência nas áreas em questão. Entretanto, a hipótese da existência de $A$. guariba em Fênix não pode ser completamente descartada.

Pseudalopex gymnocercus (G. Fischer, 1814) (Canidae): a distribuição deste carnívoro está limitada quase que exclusivamente aos Campos Naturais (Margarido \& Braga 2004). A escassez de citações nas entrevistas $(n=2)$ denota o caráter duvidoso, mas não improvável, de sua ocorrência na região.

Revista Brasileira de Zoologia 22 (4): 991-1002, dezembro 2005 
As informações recentes sobre outras espécies de ambientes abertos que vêm ampliando sua distribuição geográfica dado à transformação de áreas florestais em áreas de pastagem e plantações (STraube 1998, CÁCERes 2004) reforçam a necessidade de investigações nesse sentido para área de estudo.

Leopardus wiedii: todos os registros deste felídeo foram realizados em formações florestais (MARGarido \& Braga 2004). A dificuldade para leigos em diferenciar L. tigrinus de L. wiedii pode ser a razão pela qual a espécie só tenha sido citada em uma entrevista. Entretanto, sua ocorrência na área foi comprovada por visualização de dois indivíduos na FC predando morcegos capturados em redes-de-neblina.

Pteronura brasiliensis: a distribuição original da ariranha estendia-se por todo território brasileiro. Contudo, no Paraná, poucos são os relatos de sua ocorrência, sendo um deles para o rio Ivaí, no final do século XIX (BIGG-Wither 1974, Muricy 1975). Atualmente, a espécie conta com uma pequena população que ocupa uma área de Floresta Estacional Semidecidual no rio Paraná, tendo desaparecido nas demais bacias hidrográficas (MARGARIDO \& BRAGA 2004). Sua presença para a área de estudo foi relatada em quase $70 \%$ das entrevistas, sendo que em muitas delas houve a confirmação da existência do animal na região ainda em dias atuais. Tal informação deve ser investigada, já que possibilita a descoberta de uma segunda população da espécie para o estado.

Conepatus chinga (Molina, 1782) (Mustelidae): sua presença no Paraná é baseada em um único registro, realizado na Floresta Ombrófila Densa Submontana, na Área de Especial Interesse Turístico do Marumbi (CÁceres 2004). Para a área de estudo foi relatada somente por um entrevistado, podendo ser decorrente da má identificação da fotografia apresentada, embora a citação do odor característico proceda. O forte odor do zorrilho, relatado pelos entrevistados, provém de secreções produzidas por glândulas peri-anais, com função de defesa (Mondolfi 1973).

Tapirus terrestris: a área original de ocorrência desta espécie era praticamente todo o Paraná; atualmente seus registros são raros, dado o desaparecimento das florestas (MARGARIDO \& Braga 2004). A presença atual da anta em Fênix é duvidosa mesmo para os entrevistados, que relataram a última visualização para o ano de 1972. Entretanto, em julho de 1995, foi encontrado uma amostra fecal da espécie no VR, às margens do rio Corumbataí (S.B. Mikich, obs. pess.). Provavelmente este animal chegou no VR pelo rio, vindo de remanescentes florestais relativamente próximos (aproximadamente $20 \mathrm{~km}$ ). Esta foi a última evidência de T. terrestris para a área de estudo.

Mazama nana e M. gouazoupira: a ocorrência de ambas espécies abrange praticamente todo o Paraná (MARgarido \& BragA 2004). A identificação específica do gênero Mazama com base na morfologia externa é bastante difícil (DuARTE 1997), podendo ser esta a razão pela qual, somente M. americana $\mathrm{e}$ Mazama sp. haviam sido registradas para a área de estudo por meio de evidências diretas. No entanto, baseado em informações obtidas junto aos entrevistados, como os nomes populares e a existência de três espécies distintas (apresentadas ao decorrer deste trabalho), M. nana e M. gouazoupira foram consideradas para a região.

Pecari tajacu: a distribuição original desta espécie, que se estendia por todo o estado, hoje está restrita a poucas regiões (Margarido \& Braga 2004). Para a área de estudo, embora a espécie tenha sido citada em mais de $50 \%$ das entrevistas, todos a consideraram localmente extinta, tendo seus últimos registros em meados da década de 1990. No entanto, há registros atuais para um remanescente florestal localizado no município de São Pedro do Ivaí (Margarido \& Braga 2004), na margem direta do rio Ivaí, distando aproximadamente $5,7 \mathrm{~km}$ em linha reta da FC e $10 \mathrm{~km}$ do VR.

Como visto acima, seis táxons podem ser considerados como novos e atuais registros para Fênix: C. tatouay, E. sexcinctus, M. tridactyla, L. wiedii, M. nana e M. gouazoupira. Adicionalmente, a presença de $T$. terrestris e $P$. tajacu pode ocorrer de maneira esporádica, frente ao deslocamento de indivíduos de remanescentes florestais próximos àqueles localizados em Fênix. No entanto, a permanência dessas espécies nas áreas de estudo deve ser dificultada pelas pequenas dimensões dos fragmentos florestais e pelas pressões de caça diagnosticadas.

O número de mamíferos para a região pode ser ampliado, se investigações sobre espécies duvidosas (C. minimus, D. septemcinctus, A. guariba e $P$. brasiliensis) forem realizadas e suas presenças confirmadas. Sendo assim, apenas $C$. chinga e $P$. gymnocercus são de ocorrência improvável para área de estudo.

Dentre as espécies tidas como constantes, ou seja, as mais citadas nas entrevistas, 53\% pertencem à Ordem Carnivora. Isto pode ser conseqüência do fato deste grupo ser freqüentemente visto com fascínio e admiração, representando também uma fonte de perigo ao homem e aos rebanhos domésticos (SCHALler 1998). Além dos carnívoros, as espécies mais citadas foram aquelas de interesse cinegético (veados, porcos-do-mato, grandes roedores e tatus), o que pode ser explicado pelo fato das entrevistas terem sido realizadas, na maioria das vezes, com pessoas que utilizam a caça como fonte de renda e alimento e/ou que viveram em uma época em que esta atividade era permitida e amplamente realizada.

A respeito dos nomes populares, algumas espécies merecem destaque por apresentarem determinadas variações locais em relação aos nomes disponíveis na literatura. Herpailurus yaguarondi possui uma grande quantidade de nomes populares no território brasileiro, tais como gato-mourisco, gato-vermelho, gato-preto, maracajá-una e jaguarundi (FonseCA et al. 1994, Rodrigues \& Auricchio 1994a, Silva 1994, Oliveira \& Cassaro 1999). Embora entre os entrevistados, a designação mourisco tenha sido a mais utilizada, outros nomes também foram mencionados, como: mourício (provavelmente uma variação de mourisco) e canguçu, que é comumente empregado para designar Panthera onca (Oliveira \& Cassaro 1999). Os gatos-do-

Revista Brasileira de Zoologia 22 (4): 991-1002, dezembro 2005 
mato L. tigrinus e L. wiedii também foram chamados de "mamoninha" por um dos entrevistados, nome que, até então, não constava na literatura para estas espécies.

Galictis cuja, tradicionalmente conhecido por furão (FonSECA et al. 1994, Rodrigues \& AURICCHIO 1994b, SiLva 1994), é também chamado localmente de jaratataca e guaripeva. Os nomes jaratataca ou jaritataca normalmente são atribuídos às espécies do gênero Conepatus (Emmons 1997, Margarido \& Braga 2004). O fato dos animais destes dois gêneros possuírem como característica o forte odor exalado no momento em que se encontram sob ameaça, pode ter levado a uma identificação errônea. Não foram obtidas citações nem aplicações do nome guaripeva na literatura consultada.

Para $P$. cancrivorus, nomes como mão-pelada e guaxinim são os mais conhecidos e difundidos na literatura, entretanto, os nomes quatizão e guará devem ser considerados variações regionais. Nasua nasua é denominado mundialmente como quati ou coati (Fonseca et al. 1994, Rodrigues \& AURICCHIO 1994c, SILVA 1994); a atribuição do nome quati-mundéo é feita para exemplares de maior porte, normalmente machos que vivem solitários ou em grupos de dois ou três indivíduos (CABRERA \& Yepes 1960, Schweizer 1992).

Para os veados do gênero Mazama os nomes populares não apresentaram inovações em relação aos disponíveis na literatura. Veado-mateiro, veado-vermelho, veado-pardo e guatapará normalmente são atribuídos à M. americana (CIMARDI 1996, Duarte 1996, Fonseca et al. 1996, Miranda \& Mangabeira 2002). Segundo Margarido \& Braga (2004), o nome cambuta é aplicado, no Paraná, à espécie $M$. nana, e o nome veadocatingueiro é utilizado apenas para M. gouazoupira (DuARte 1996, Fonseca et al. 1996, EMmons 1997, Margarido \& BRAga 2004).

\section{Sobre a caça}

Ainda que apenas 26\% dos entrevistados tenham admitido a realização da caça, essa atividade parece ser uma prática comum entre os moradores de Fênix, especialmente pelo fato de $84 \%$ dos entrevistados admitir já ter se alimentado de animais silvestres.

A exemplo de toda a região neotropical, as espécies de maior interesse cinegético na área de estudo foram os porcosdo-mato, veados, antas, grandes roedores e tatus (REDFord \& Robinson 1987, Bodmer et al. 1997, Cullen Jr. et al. 2000). Amplamente citada nas entrevistas, a acentuada caça da capivara, nos dias de hoje, pode ser explicada pela presença deste grande roedor em muitas áreas não protegidas.

As espécies que sofrem maior pressão de caça na região, assim como em todo neotrópico (JoRGENSON \& REDFORD 1993, Leite \& Galvão 2002), são também as principais presas dos grandes felinos (Puma concolor (Linnaeus, 1771) (Felidae) e Panthera onca). Deste modo, os humanos passam a ser competidores diretos por recursos alimentares com os carnívoros, podendo afetar negativamente suas populações e levá-las ao declínio, já acentuado por fatores como a caça direta e a destruição dos seus hábitats naturais.
Com relação às localidades ainda utilizadas para a caça, o VR continua sendo a área mais procurada, mesmo se tratando de uma unidade de conservação de proteção integral, onde há fiscalização permanente e a visitação é controlada (МıкıcH \& Oliveira 2003).

Cullen JR. et al. (2000), em uma análise comparativa dos efeitos da caça em vários fragmentos de Floresta Estacional Semidecidual, relataram que a abundância de Pecari tajacu, Agouti paca e primatas não mostrou diferença frente esta pressão, enquanto que Tapirus terrestris, Tayassu pecari, tatus e Nasua nasua sofreram reduções em sua abundância. Com exceção de $N$. nasua, que ainda possui populações relativamente grandes na região, tais resultados parecem também se aplicar aos remanescentes abordados por este estudo. Em complemento, CulLEN JR. et al. (2000) sugerem ainda que, em áreas fragmentadas onde a pressão de caça é intensa, os efeitos da fragmentação são potencializados, e são provavelmente os mais importantes fatores da extinção de espécies como T. terrestris e T. pecari. Tais fatos podem justificar a extinção destas espécies em Fênix.

O Paraná não possui informações disponíveis sobre o comércio ilegal de peles e couro de animais silvestres. Deste modo, os dados contidos neste trabalho, indicando as espécies mais procuradas na região com este propósito (Leopardus spp., Herpailurus yaguarondi, Tayassu pecari, Pecari tajacu, Mazama spp. e Chironectes minimus), são de grande relevância para a sua conservação no estado.

\section{Ataques a criações domésticas}

Normalmente, os carnívoros silvestres não possuem o hábito de atacar criações domésticas, já que em ambientes que apresentam condições para a sua sobrevivência, estes animais evitam qualquer contato com o homem e suas criações. Entretanto, devido à diminuição das suas presas naturais em virtude da caça predatória e da fragmentação do hábitat, os carnívoros podem atacar espécies domésticas (AzEvedo \& Conforti 2002).

Assim como o observado neste trabalho, a predação de animais domésticos realizada por espécies de carnívoros que não a onça-pintada e o puma, geralmente ocorrem sobre galinhas e patos (Oliveira \& Cavalcanti 2002). Pelo fato de suas presas serem pequenas, dificultando o encontro das carcaças, é comum a espécie responsável pelo ataque ser identificada com base nos rastros deixados (Oliveira \& Cavalcanti 2002). Entretanto, em Fênix, também foram citadas outras técnicas de identificação dos predadores, como a sondagem, a captura com armadilhas e o abate do animal.

Embora alguns estudos tenham sido realizados no Brasil em relação aos ataques de criações domésticas, como bovinos e ovinos, por Panthera onca e Puma concolor (e.g. Quigley \& Crawshaw Jr. 1992, Crawshaw Jr \& Quigley 2002, Mazzolli et al. 2002), informações sobre a freqüência de ataque e os prejuízos causados pelos demais carnívoros são pouco reportados. Em outros países da América do Sul, como o Uruguai, existe uma maior preocupação com a predação causada por carnívoros de menor porte, como os canídeos silvestres sobre as criações de 
ovinos. Como exemplo, Cravino et al. (2000) relataram a presença de carneiros em 14,3\% da dieta de Cerdocyon thous e 20,8\% da dieta de Pseudalopex gymnocercus; no entanto, estes predadores só foram responsáveis pela morte de $0,4 \%$ dos ovinos recém-nascidos. Este tipo de informação vem a ser de grande relevância para subsidiar propostas de manejo e medidas de prevenção e controle de danos aos produtores.

Mesmo a população local estando ciente de que seus animais domésticos estão sendo predados principalmente pela forma (extensiva) como são criados, é necessária a realização de trabalhos mais intensivos de educação ambiental e de orientação dos moradores dos arredores das áreas florestadas da região. Assim, novos casos de ataque poderão ser evitados, uma vez que eles geralmente acontecem nas proximidades de unidades de conservação e outros remanescentes florestais (AzEvEDO \& CONFORTi 2002). A falta de uma política regional para o manejo das criações, que vise a diminuição da freqüência da predação por carnívoros, faz com que os proprietários rurais resolvam este problema, muitas vezes, eliminando os predadores (Oliveira \& Cavalcanti 2002), diminuindo suas populações, muitas delas já ameaçadas. A utilização de cachorros domésticos para evitar os ataques às criações é considerada uma prática eficaz, segundo os próprios entrevistados. Entretanto, esses cachorros muitas vezes acabam por matar os carnívoros silvestres em tentativas de predação ou quando perseguidos dentro dos fragmentos florestais.

\section{Alterações temporais na composição da mastofauna}

A perda de hábitat e a caça têm sido consideradas as principais razões da diminuição das populações e da extinção de espécies (REDFord 1997). De fato, 74\% dos entrevistados relacionaram-nas como as principais causas das alterações na composição da mastofauna de Fênix. Entretanto, também é considerada como causa de alterações na composição mastofaunística uma severa tempestade que ocorreu na região em outubro de 1995. Durante esta catástrofe, um vento devastador, formando uma espécie de ciclone, derrubou árvores de até 30 metros de altura, destruindo também o subosque do VR; houve, ainda, uma chuva de granizo tão intensa que desfolhou a maioria das plantas arbóreas e arbustivas. Como conseqüência foi notada a ausência total de frutos nos meses de outubro e novembro deste mesmo ano; o que provavelmente afetou negativamente as populações dos animais frugívoros, principalmente os especialistas (MiкICH 2003).

O fato de $21 \%$ dos entrevistados acreditarem que atualmente exista um maior número de mamíferos na região pode estar relacionada ao aumento da população de espécies como Cebus nigritus e Nasua nasua (Мıкісн \& Oliveira 2003), informação confirmada por $50 \%$ dos entrevistados.

A explosão populacional de algumas espécies de predadores de médio porte como resultado, principalmente, da perda de seus predadores naturais, é um problema comum em muitos fragmentos florestais, sobretudo em áreas reduzidas e isoladas (Wilcove 1985). Segundo Мiкich \& Oliveira (2003), o
VR é um exemplo deste fenômeno, pois dada a sua pequena área e a matriz que o cerca (monoculturas), não oferece mais as condições necessárias para o suporte de grandes predadores, como P. onca, P. concolor e Harpia harpyja (Linnaeus, 1758) (Aves: Accipitridae), favorecendo, entretanto, o crescimento descontrolado de populações de mamíferos de médio porte e hábito alimentar oportunista, como o N. nasua e C. nigritus (VIDolin \& Мiкich 2004). Diferentemente do que foi dito em parte das entrevistas, T. pecari, mesmo se alimentando das plantações, não tem aumentado sua densidade no VR; de fato, o contrário tem sido observado (S.B. Mikich, obs. pess.).

\section{CONSIDERAÇÕES FINAIS}

As abordagens etnozoológicas apresentadas neste trabalho foram efetivamente substanciais à obtenção de informações históricas e atuais sobre a mastofauna regional, incluindo seus aspectos bio-ecológicos. De fato, quando observado o acréscimo de espécies à lista mastofaunística pré-existente, fica claro seu valor como ferramenta para estudos de inventário.

Os dados obtidos quanto às extinções locais de mamíferos de grande porte, aspectos relacionados à caça, predações causadas às criações domésticas por carnívoros selvagens e alterações na composição mastofaunística local, demonstram a eficiência do método no diagnóstico de problemas relacionados à conservação da fauna, incluindo, até mesmo, um componente temporal.

Embora exista, obviamente, a necessidade de investigar mais detalhadamente algumas das informações obtidas junto à população local antes que sua veracidade possa ser atestada, os dados aqui relatados são uma importante fonte de embasamento e direcionamento para ações conservacionistas a serem desenvolvidas na região, além de contribuir significativamente para o aumento do conhecimento da mastofauna paranaense.

\section{AGRADECIMENTOS}

Ao Projeto "O Parque Estadual de Vila Rica e a conservação da biodiversidade no Paraná": Instituto Ambiental do Paraná (IAP), Secretaria do Meio Ambiente do Estado do Paraná (SEMA), Fundo Nacional do Meio Ambiente (FNMA) e Ministério do Meio Ambiente (MMA), e a CAPES pelo apoio financeiro e bolsa de estudo para Fabiana Rocha Mendes. À Cláudia I. Parellada, Denise de C. Rossa Feres, Emygdio L.A. MonteiroFilho, Juliana Quadros, Lilian Casatti, Michel Miretzki e Paulo Henrique C. Marques pelas contribuições e críticas ao manuscrito.

\section{REFERÊNCIAS BIBLIOGRÁFICAS}

Azevedo, F.C.C. \& V.A. ConforTi. 2002. Fatores predisponentes à predação, p. 27-28. In: M.R.P. Leite-PitMan; T.G. Oliveira; R.C. Paula \& C. Indrusiak (Eds). Manual de identificação, prevenção e controle de predação por carnívoros. Brasília, Edições Ibama, 83p. 
Begossi, A.; R.A.M. Silvano; B.D. do Amaral \& O.T. Oyakawa. 1999. Uses of fish and game by inhabitants of an extractive reserve (Upper Juruá, Acre, Brazil). Environment, Development and Sustainability, New York, 1 (1): 73-93.

BIGG-WiTher, T.P. 1974. Novo caminho no Brasil meridional: a provincia do Parana: tres anos de vida em suas florestas e campos, 1872-1875. Rio de Janeiro, J. Olympio, 417p.

Bodmer, R.E.; J.F. EISENBERg \& K.H. REDFord. 1997. Hunting and the likelihood of extinction of Amazonian mammals. Conservation Biology, Boston, 11 (2): 460-466.

Cabrera, A. \& J. Yepes. 1960. Mamíferos sud-americanos. Vida, costumbres y descripción. Buenos Aires, Comp. Argent. Edit., $2^{\mathrm{a}}$ ed., $370 \mathrm{p}$.

CÁceres, N. 2004. Ocorrence of Conepatus chinga (Molina) (Mammalia, Carnivora, Mustelidae) and other terrestrial mammals in the Serra do Mar, Paraná, Brazil. Revista Brasileira de Zoologia, Curitiba, 21 (3): 577-579.

Cimardi, A.V. 1996. Mamíferos de Santa Catarina. Florianópolis, FATMA, 302p.

Clément, D. 1998. The historical foundation of ethnobiology (1860-1899). Journal of Ethnobiology, Flagstaff, 18 (2): 161187.

Conforti, V.A. \& F.C.C. Azevedo. 2003. Local perceptions of jaguars (Panthera onca) in the Iguaçu National Park area, south Brazil. Biological Conservation, Essex, 111 (2): 215-221.

Cravino. J.L.; M.E. Calvar; J.C. Poetti; M.A. Berrutti; \& N.A. FontanA. 2000. Estudio de caso, con énfasis en la acción de "Zorros" (Mammalia: Canidae). Veterinaria, Montevideo, 35 (141): 24-44.

Crawshaw Jr., P.G. \& H.B. Quigley. 2002. Hábitos de alimentación del yaguar y del puma en Pantanal (Brasil) con las implicaciones para su gerencia y conservación, p. 223-235. In: R.A. Medellin; C. Chetkiewicz.; A. Rabinowitz; K.H. Redford; J.G. Robinson; E. SANDERSON \& A. TABER (Eds). El Jaguar en el nuevo milenio. Una evaluacion de su estado, deteccion de prioridades y recomendaciones para la conservacion de los jaguares en America. Mexico, Universidad Nacional Autonoma de Mexico, Wildlife Conservation Society, 647p.

Cullen Jr., L.; R.E. Bodmer \& C.V. PÁduA. 2000. Effects of hunting in habitat fragments of the Atlantic forests, Brazil. Biological Conservation, Essex, 95: 49-56.

Duarte, J.M.B. 1996. Guia de identificação de cervídeos brasileiros. Jaboticabal, FUNEP, 14p.

DuArte, J.M.B. 1997. Biologia e conservação dos cervídeos sul-americanos: Blastocerus, Ozotoceros e Mazama. São Paulo, FUNEP, XIV+238p.

Eisenberg, J.F. \& K.H. Redford. 1999. Mammals of the Neotropics. The Central Neotropics. Chicago,University of Chicago Press, vol. 3, X+609p.

EmMons, L.H. 1997. Neotropical rainforest mammals: a field guide. Chicago, University of Chicago Press, XVI+307p.

Ferreira, J.C.V. 1999. O Paraná e seus municípios. Cuiabá, J.C.V. Ferreira, 484p.
Fonseca G.A.B.; G. Herrmann; Y.L.R. Leite; R.A. Mittermeier; A.B. Rylands \& J.L. Patton. 1996. Lista anotada dos mamíferos do Brasil. Occasional Papers in Conservation Biology, Boston, 3: 1-38.

FonsecA, G.A.B. 2001. Proposta para um programa de Avaliação Rápida em âmbito nacional, p. 150-156. In: I. Gray \& B. DiAS (Eds). Conservação da biodiversidade em ecossistemas tropicais. Petrópolis, Editora Vozes, 430p.

Fonseca, G.A.B.; A.B. Rylands; C.M.R. Costa; R.B. Machado \& Y.L.R. LEITE. 1994. Livro vermelho dos mamíferos brasileiros ameaçados de extinção. Belo Horizonte, Biodiversitas, XX+460p.

Haverroth, M. 1997. Etnobotânica: uma revisão teórica. Antropologia em Primeira Mão, Florianópolis, 20: 1-56.

Iвge. 2004. Cidades. Disponível na World Wide Web em: http:/ /www.ibge.gov.br/cidadesat/default.php. Acesso em 22.IX.2004.

IPARDES. 2004. Leituras regionais: mesorregião geográfica centro-ocidental paranaense. Curitiba, Instituto Paranaense de Desenvolvimento Econômico e Social, 133p.

Iтсғ.1987. Plano de manejo do Parque Estadual de Vila Rica do Espírito Santo, Fênix, PR. Curitiba, Instituto de Terras Cartografia e Florestas, IX+86p.

JENSEN, A.A. 1988. Sistemas indígenas de classificação de aves: aspectos comparativos, ecológicos e evolutivos. Belém, Museu Paraense Emílio Goeldi, 88p.

Jorgenson, J.P. \& K.H. RedFord. 1993. Humans and big cats as predators in the neotropics. Symposia of the Zoological Society of London, London, 65: 637-390.

LANGE, R.B. \& E. Jablonski. 1998. Mammalia do Estado do Paraná - Marsupialia. Estudos de Biologia, Curitiba, 43: 15-224.

LeITE, M.R.P. \& F. Galvão. 2002. Yaguar, puma y pobladores locales en tres areas protegidas del bosque atlantico costero, Estado del Parana, Brasil, p. 327-259. In: R.A. Medellin; C. Chetkiewicz; A. Rabinowitz; K.H. Redford; J.G. Robinson; E. SANDERSON \& A. TABER. (Eds). El Jaguar en el nuevo milenio. Una evaluacion de su estado, deteccion de prioridades y recomendaciones para la conservacion de los jaguares en America. Mexico, Universidad Nacional Autonoma de Mexico, Wildlife Conservation Society, 647p.

MAACK, R. 1981. Geografia física do Estado do Paraná. Rio de Janeiro, J.Olympio, Secretaria da Cultura e do Esporte do Governo do Estado do Paraná, XLIII+442p.

Margarido, T.C.C \& F.G. Braga. 2004. Mamíferos, p. 27-142. In: S.B. Мiкich \& R.S. BÉRnils (Eds). Livro vermelho da fauna ameaçada no Estado do Paraná. Curitiba, Instituto Ambiental do Paraná, XVI+763p.

Martin, G.J. 1995. Ethnobotany, a methods manual. London, Chapman \& Hall, 268p.

Mazzolli, M.; M.E. Graipel \& N. Dunstone. 2002. Mountain lion depredation in southern Brazil. Biological Conservation, Essex, 105: 43-51.

Mirich, S.B. \& K.L. Oliveira. 2003. Revisão do plano de mane- 
jo do Parque Estadual Vila Rica do Espírito Santo. Mater Natura. Curitiba, Instituto de Estudos Ambientais, Ministério do Meio Ambiente e Fundo Nacional do meio Ambiente, XXI+452p.

Мıкıch, S.B. \& S.M. SıIVA. 2001. Composição florística e fenologia das espécies zoocóricas de remanescentes de Floresta Estacional Semidecidual no centro-oeste do Paraná, Brasil. Acta Botânica Brasilica, Porto Alegre, 15 (1): 89-113.

Мıкісн, S.B. 2003. Efeitos de uma tempestade tropical sobre a disponibilidade de frutos zoocóricos e seus consumidores em um remanescente de Floresta Estacional Semidecidual do Paraná, Brasil. Anais do VI Congresso de Ecologia do Brasil, Fortaleza, p.588-590.

Мiкich, S.B.; S.M. Silva \& M.M. BRiтTo. 2004. O projeto Malha Florestal e o papel das unidades de conservação na manutenção da Floresta Estacional Semidecidual no Estado do Paraná, Brasil, p. 260-270. In: M.S. MiLANO; L.Y. TAKAHASHI \& M.L. NunEs (Eds). Anais IV Congresso Brasileiro de Unidades de Conservação, vol. 1, Curitiba, 736p.

Miranda, J.B. \& J.A.C. Mangabeira. 2002. Extrativismo animal em zona de fronteira agrícola na Amazônia, o caso do município de Machadinho d'Oeste - RO. Campinas, Embrapa Monitoramento por Satélite, 36p.

Mondolfi, E. 1973. El mapurite, um animal beneficioso. Defensa de la Naturaleza, Caracas, 1 (1): 31-35.

Muricy, J.C. 1975. Viagem ao país dos jesuítas. Curitiba, Imprensa Oficial do Estado do Paraná, 406p.

Oliveira, T.G. \& K. Cassaro. 1999. Guia de identificação dos felinos brasileiros. São Paulo, Sociedade de Zoológicos Brasileiros, $2^{\mathrm{a}}$ ed., $60 \mathrm{p}$.

Oliveira, T.G. \& S.M.C. Cavalcanti. 2002. Identificação de predadores de animais domésticos, p. 31-50. In: M.R.P. LeITEPitman; T.G. Oliveira; R.C. Paula \& C. Indrusiak (Eds). Manual de identificação, prevenção e controle de predação por carnívoros. Brasília, Edições Ibama, 83p.

Paranacidade. 2004. Fênix. Disponível na World Wide Web em: http://www.paranacidade.org.br/municipios/ municipio.php. Acesso em 01.IX.2004.

Pardini, R.; E.H. DitT; L. Cullen Jr.; C. Bassi \& R. Rudran. 2003. Levantamento rápido de mamíferos de médio e grande porte, p. 181-201. In: L. Cullen Jr.; R. Rudran \& C. ValladaresPAdua (Eds). Métodos de estudos em biologia da conservação e manejo da vida silvestre. Curitiba, Editora UFPR, $665 \mathrm{p}$.

Parellada, C.I. 1993. Villa Rica del Espiritu Santo: ruínas de uma cidade colonial espanhola no interior do Paraná. Arquivo do Museu Paranaense, Nova série, Arqueologia, Curitiba, 8: 1-58.

Pedroso JR., N.N. \& M. SAto. 2003. Percepção de fauna terrestre e conservação no Parque Nacional do Superagui. Revista de Educação Pública, Cuiabá, 12 (21): 43-70.

Quigley, H.B. \& P. Crawshaw JR.. 1992. A conservation plan for the jaguar Panthera onca in the Pantanal region of Brazil.
Biological Conservation, Essex, 61: 149-157.

RedFord, K.H. \& J. Robinson. 1987. Hunting by Indigenous Peoples and Conservation of Game Species. Cultural Survival Quarterly, Cambridge 9 (1): 41-44.

Redford, K.H. 1997. A floresta vazia, p.1-22. In: C. ValladaresPADUA \& Bodmer, R.E. (Eds). Manejo e conservação de vida silvestre no Brasil. Mamirauá, Sociedade Civil, X+283p.

Rodrigues, A.S.M \& P. Auricchio. 1994a. Felinos do Brasil. São Paulo, Coleção Terra Brasilis, Série Zoologica - Zoo II, Mamíferos do Brasil, 17p.

Rodrigues, A.S.M \& P. Auricchio. 1994b. Mustelídeos do Brasil. São Paulo, Coleção Terra Brasilis, Série Zoologica - Zoo III, Mamíferos do Brasil, 13p.

Rodrigues, A.S.M \& P. Auricchio. 1994c. Procionídeos do Brasil. São Paulo, Coleção Terra Brasilis, Série Zoologica - Zoo IV, Mamíferos do Brasil, 7p.

SCHALLER, G.B. 1998. Introduction: carnivores and conservation biology, p. 1-10. In: J.L. GiTTLEMAN (Ed.). Carnivore, behavior, ecology and evolution. London, Cornell University Press, vol. 2, 640p.

Schweizer J. 1992. Ariranhas no pantanal: ecologia e comportamento da Pteronura brasiliensis. Curitiba, Editora Brasil Natureza Ltda, 202p.

Silva, F. 1994. Mamíferos Silvestres - Rio Grande do Sul. Porto Alegre, Fundação Zoobotânica do Rio Grande do Sul, $2^{\text {a }}$ ed., $246 \mathrm{p}$.

Silveira-Neto, S.; O. Nakano; D. Barbin \& N.A.V. Nova. 1976. Manual de ecologia dos insetos. São Paulo, Editora Agronômica Ceres, 419p.

Straube, F.C. 1998. O cerrado no Paraná: ocorrência original e atual e subsídios para sua conservação. Cadernos da Biodiversidade, Curitiba, 1: 12-24.

Veloso, H.P.; A.L. Rangel Filho \& J.C.A. Lima. 1991. Classificação da vegetação brasileira, adaptada a um sistema universal. Rio de Janeiro, IBGE, 124p.

Vidolin, G.P \& S.B. MıкICH. 2004. Cebus nigritus (Primates: Cebidae) no Parque Estadual Vila Rica do Espírito Santo, Fênix - PR: Estimativa populacional e área de vida, composição e dinâmica de grupos, p. 196-205. In: M.S. Milano; L.Y. TAKAHASHI \& M.L. Nunes (Eds). Anais IV Congresso Brasileiro de Unidades de Conservação. Curitiba, vol. 1, 736p.

Vidolin, G.P.; T. UchôA; P.R. Mangini; F.R. Mendes; A.M. Kuczach \& N.G.C. Borlachenco. 2002. Felinos na Reserva Natural Salto Morato, Guaraqueçaba, Paraná; Brasil: Levantamento e caracterização de seus aspectos ecológicos, p. 804-812. In: M.S. Milano \& V. Theulen (Eds). Anais II Congresso Brasileiro de Unidades de Conservação. Campo Grande, vol. 2, 845p.

Voss, R.S. \& L.H. Emmons. 1996. Mammalian diversity in neotropical lowland rainforests: a preliminary assessment. Bulletin of the American Museum of Natural History, New York, 230: 1-115.

Wilcove, D.S. 1985. Nest predation in Forest tracts and the decline of migratory songbirds. Ecology, Tempe, 66 (4): 1221- 
1214.

Wilson, D.E. \& D.M. Reeder. 1993. Mammal Species of the World: a taxonomic and geographic reference. Washington, Smithsonian Institution Press, $2^{\text {nd }}$ ed., 1206p.

Wilson, D.E.; C.F. Ascorra \& S. Solari. 1996. Bats as indicators of habitat disturbance, p. 613-625. In: D.E. WiLson \& A. SONDOVAL (Eds). Manu: The biodiversidade of southeastern Peru. Washington, National Museum of Natural History, Smithsonian Institution, Office of Biodiversity Programs, 679 p.

Recebido em 07.III.2005; aceito em 02.XI.2005. 